அறபுமொழியிலிருந்து தமிழுக்கு

மொழிபெயர்ப்புச் செய்வதிலுள்ள கலாசாரச் சிக்கல்கள்

பீ. எம். ஹம்தூன் அ, •

அ அறபியல்துறை, கிழக்குப்பல்கலைக் கழகம், வந்தாறுமூலை, இலங்கை.

\title{
Cultural Issues in Translating from Arabic to Tamil
}

\section{P.M. Hamthoon a, *}

a Department of Arabic, Faculty of Arts \& Culture, Eastern University, Sri Lanka.

* Corresponding Author: hamthoonpms@esn.ac.lk

Received: 18-09-2021 Revised: 24-01-2022

Accepted: 26-01-2022

Published: 30-01-2022

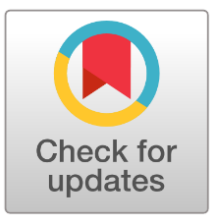

\begin{abstract}
Translation has been used by every community in the world throughout the history as a tool to derive many new things from other language and cultural backgrounds that are not present in the particular culture. In Islamic history during the reign of the Umayyad, the treasuries of knowledge from Greek territory were transslated into Arabic. This is what led to the formation of modern Europe and the scientific, industrial and technological revolution in the world. Tamil-speaking Muslims living in the Indian subcontinent, especially in countries such as India and Sri Lanka, were interested in translating religious texts from Arabic to Tamil, including the Qur'an and Al-Hadith literatures, the main sources of Islam. Thus, during the translation process that took place between languages belonging to different language families, translation faced various challenges. Particularly, Translators have had some cultural problems. Those translators also tried to solve it. This study has found the need to build culture in translation, the importance of translation in maintaining inter-cultural communication and the possible ways to identify and resolve issues that arise when translating from Arabic to Tamil.
\end{abstract}

Keywords: Cultural Problem, Translation, Arabic, Tamil

\section{முன்னுரை}

மொழி என்பது நாம் பிறருடன் தொடர்பு கொள்ள பயன்படுத்தும் ஒரு வழிமுறையாகும். இது கலாசாரம், சமூகம் மற்றும் நம்பிக்கைகளின் வெளிப்பாடாக அமையக் கூடியது. உலகெங்கிலும் ஆயிரக்கணக்கான கலாசாரங்கள் உள்ளன. அவை அனைத்தும் பிரத்தியேக விசயங்களை அந்தந்த மொழிகள் மூலம் வெளிப்படுத்துகின்றன. பிறமொழியொன்றில் உருவாகும் இலக்கிய படைப்பொன்றை மொழிபெயர்ப்பின் வாயிலாகவே மற்ற மொழியினரால் புரிந்து கொள்ள முடிகின்றது.

“மொழி ஒரு தொடர்பாடல் ஊடகம். ஒருவரது சிந்தனையையும் மனவோட்டத்தையும் எண்ணங்களையும் சரிவர வெளிக்காட்ட மொழி உதவுகிறது. குறிப்பிட்டவொரு மொழியைப் பேசுவோரிடையே கருத்துப்பரிமாற்றத்தை செய்ய மொழி முக்கிய பங்காற்றினாலும் ெவ்வேறு மொழிகளைப் பேசும் சமூகங்களிடையே கருத்துப் பரிமாற்றத்தைச் செய்ய மொழிபெயர்ப்பே உதவுகிறது. உணர்வுகளைப் பரிமாறிக் கொள்வதில் மொழிபெயர்ப்பு முக்கிய பங்காற்றுகிறது" (Mohamed Ashraff \& Ibrahim, 2013). 
ஒரு மொழியிலிருந்து மற்றொரு மொழிக்கு வார்த்தைகளை மாற்றுவதை விடவும் மொழிபெயர்ப்பு கடினமானது. அது வார்த்தைகளையன்றி காலாசாரங்களுக்கிடையில் உறவினைக் கட்டியெழுப்புகின்றது. தனது சொந்த மொழி மற்றும் கலாசார பின்புலங்களுக் கூடாக கற்றுக் கொள்ள முடியாத பல விசயங்களை கற்றுக் கொள்ளவும், அடைய முடியாத உலகின் பல அறிவியல் படைப்புக்களைச் சென்றடையவும் மொழிபெயர்ப்பு உதவுகின்றது.

“மொழிபெயர்ப்பு என்பது மனித சிந்தனைகளையும் கருத்துக்களையும் பரிமாற்றம் செய்வதற்கான ஒரு மனித செயற்பாடாகும். மொழியை மையமாகக் கொண்ட சமூகங்களுக்கிடையில் இணைப்புப் பாலமாக மொழிபெயர்ப்பு உள்ளது. மொழிபெயர்ப்பினூடாக சமூக, கலாசார, மொழியியல் ரீதியான பெறுமானங்கள் ஒரு மொழியிலிருந்து மற்றொரு மொழிக்கு கொண்டு செல்லப்படுகின்றன. அது ஒரு ஆக்கபூர்வமான செயற்பாடு. அடிப்படை மூலத்திலிருந்து மாறிச் செல்லாமல் ஆக்கபூர்வமானதாக உருவாக்கத்திறன் மொழிபெயர்ப்புக்கு அவசியம். ஒரு சிறந்த மொழிபெயர்ப்பு மூலத்தின் கருத்துக்கள், கட்டமைப்பு மற்றும் கலாசார அம்சங்களை முழுமையாகக் கொண்டிருக்கும்" (Ashraff, 2010).

அடிப்படையில் மொழிபெயர்ப்பு மனித சமூக நடத்தையுடன் தொடர்புபட்ட ஒன்று என்பதனால் அது பெரும்பாலும் மனித பண்பாட்டு கலாசார அம்சங்களுடன் கலந்து விட்டது. இது மனித வாழ்விலிருந்து பிரிக்க முடியாத கலாசாரக் கூறாகவும் உள்ளது. "மனித அறிவு வளர்ச்சி வெளிப்பாட்டில் மொழிபெயர்ப்பு எனும் துறை அறிவியல் அடிப்படையில் இயங்கும் கூறாகும். மூலமொழியில் (Source language) உள்ள ஒரு பகுதியை இலக்குமொழியில் (Target language) கருத்து மாறாமல் மொழிமாற்றம் செய்வது மொழிபெயர்ப்பு ஆகும். மூலத்திற்கு இணையான பெறுமொழி நிகரனை (equivalent) கண்டறியும் முயற்சியே மொழிபெயர்ப்பு என்பார் ஐசடோப் பிஞ்சக். ஒரு மூலப்பிரதியைப் பலர் மொழிபெயர்க்கும் போது அது ஒரே மாதிரியாக இருக்க வேண்டும். ஆனால் அப்படி அமைவதில்லை என்பது உற்றுநோக்கத்தக்கது. இந்த மொழிபெயர்ப்பு முயற்சி நடைபெறும்போது பல்வேறு சிக்கல்கள் ஏற்படுவதை நடைமுறையில் காணமுடிகிறது. மூலமொழியில் உள்ள ஒரு படைப்பு மொழிபெயர்க்கப்படும் போது இலக்குமொழியில் அந்த படிகள் ஒரே தன்மை கொண்டதாக இருக்க வாய்ப்பில்லை. இதனால் மூலமொழி ஆசிரியரின் ஆளுமையும் மொழிபெயர்ப்பாளனின் திறனும் கேள்விக்குறியாகிவிடுகிறது. இதற்குக் காரணம் மொழிபெயர்ப்பாளனின் நேர்மை, மொழிவளம், சொல்தேர்வு, இலக்கியப்புலமை, பண்பாட்டு மரபுத்தாக்கம், துறைசார்ந்த கலைச்சொல்லாக்க அறிவு முதலிய காரணிகள் அடிப்படையாக அமைகிறது" (Chithambaram, 2019).

\section{ஆய்வு நோக்கம்}

இவ்வாய்வு பின்வரும் நோக்கங்களைக் கொண்டுள்ளது:

1. மொழிபெயர்ப்பின் முக்கியத்துவத்தை அறிமுகம் செய்தல்.

2. மொழிபெயர்ப்புக்கும், கலாசாரத்திற்குமிடையிலான தொடர்பினைக் கண்டறிதல்

3. அறபு மொழியிலிருந்து தமிழுக்கு மொழிபெயர்ப்புச் செய்யும் போது ஏற்படும் கலாசாரச் சிக்கல்களை இனங்கண்டு தீர்த்தல்.

\section{ஆய்வு முறையியல்}

ஆய்வு நோக்கங்களை அடைந்து கொள்வதற்காக விவரண மற்றும் பகுப்பாய்வியல் முறைகள் பயன்படுத்தப்பட்டன. முதலாம் நிலைத்தரவுகளுடன் இரண்டாம் நிலைத்தரவுகளாக நூல்கள், சஞ்சிகைள், பத்திரிகைகள் மற்றும் இணையம் போன்றவற்றின் மூலம் தரவுகள் பெறப்பட்டு ஆய்வுக்குட்படுத்தப்பட்டன. 


\section{பெறுபேறுகளும் கலந்துரையாடலும்}

\section{மொழியெர்ப்பும் இஸ்லாமியரும்}

மனித வரலாற்றில் தோன்றி வளர்ந்த எல்லா நாகரிகங்களும் மொழிபெயர்ப்பில் ஈடுபட்டுள்ளனர். இஸ்லாமிய மக்களும் இதற்கு விதி விலக்கானவர்கள் அல்லர். பாரம்பரிய அறபு முஸ்லிம்கள் பல கலாசார பின்புலங்களைக் கொண்ட அறிவுப் படைப்புக்களை தமது மொழிக்கு கொண்டு வந்தனர். “கிறிஸ்து ஆண்டின் ஆரம்பத்தில் அறேபியக் கல்வியின் வளர்ச்சியின் காரணமாகக் கிரேக்க மொழியிலிருந்து ஏராளமான மொழிபெயர்ப்புக்கள் தோன்றின. அரிஸ்டாட்டில், ்பேட்டோ போன்றோருடைய படைப்புக்கள் அரேபிய மொழியில் மொழிபெயர்க்கப்பட்டன. எட்டாம் நூற்றாண்டில் ஸ்பெயின் நாட்டின் மீீு மூரிஷ் படையெடுத்தது. அதன் காரணமாக அரேபிய மொழிப் புத்தகங்கள் லத்தீன் மொழிக்கு மொழி மாற்றம் செய்யப்பட்டன. பன்னிரண்டாம் நூற்றாண்டில் டொலடோ என்னுமிடத்தில் ஜெரோட் ஆப் கிரோமனா கிரேக்க அரேபிய மொழியிலுள்ள படைப்புக்களை லத்தீன் மொழிக்கு மொழி மாற்றம் செய்தார்" (Vealyutham,1985).

\section{இஸ்லாமிய இலக்கியங்களை மொழிபெயர்க்க வேண்டியதன் தேவைப்பாடு}

சிந்துப் பிரதேசங்களில் இஸ்லாமியப் பரவலின் பின்னர், அறபு மொழிப் படைப்புக்கள் இந்திய திராவிட மொழிகளில் மொழிபெயர்க்கப்படத் தொடங்கின. 1880களில் திருக்குர்ஆன் மொழிபெயர்க்கப்பட்டது. அதனைத் தொடர்ந்து ஏனைய அறபு மொழிப் பொக்கிஷங்களும் மெல்ல மெல்ல தமிழ் மொழிக்கு கொண்டு வரப்பட்டன. இது அறபு மொழிப் பரிச்சயம் இல்லாத மக்கள் இஸ்லாமியப் போதனைகளை புரிந்து கொள்ள வேண்டுமென்ற நோக்கில் செய்யப்பட்டது. "தற்காலத்தில் மொழிகள் மற்றும் இலக்கியங்களுக்கிடையில் ஒப்பியல் செயற்பாடு இடம்பெறுகின்றது. இதனால், மனிதன் மொழியைக் கற்பதன் மூலம் அல்லது மொழிபெயர்ப்பு மூலம் பன்னாட்டு மொழிகளிலுள்ள படைப்பாற்றல்களை அறிந்துகொள்ள விரும்புகின்றான். அறபு மொழி உலக மொழிகளுள் உயர்ந்த இடத்தினைப் பெற்றதாலும், அதன் இலக்கியங்கள் உயிரோட்டம், அழகு கொண்டு சிறப்படைவதாலும் மொழி மற்றும் இலக்கியத்துறைகளில் ஈடுபாடு கொண்டவர்கள் இந்த மொழியின் பாரம்பரியத்தையும் அதிலுள்ள பயன்மிக்க, அபூர்வமான படைப்பையும் கற்க விரும்புகின்றனர். இதற்கு மேலாக, உலகின் கண்டங்களில் ஏராளமான முஸ்லிம்கள் பரந்து வாழுகின்றனர். கிருஸ்தவத்துக்கு அ அடுத்து இரண்டாவதாக அவர்களின் சமயம் உள்ளது. எனவே, இந்த பெரும் எண்ணிக்கையினர் இஸ்லாமிய மார்க்கத்தின் வழிகாட்டல்களை அல்-குர்ஆன் மற்றும் நபிமொழியிலிருந்து தெரிந்து கொள்ளும் தேவையில் உள்ளனர். ஆனாலும், அவர்களில் பெரும்பான்மையானோர் அறபுமொழி தெரியாதவர்கள். எனவே, அல்-குர்ஆன், நபிமொழி, நபிகளாரின் வாழ்க்கைச் சரிதை (சீறா) என்பவற்றை உலக மொழிகளுக்கு மொழிபெயர்க்க வேண்டிய பெரும் தேவையுள்ளது. இதன் மூலம், அல்-குர்ஆன், சுன்னாவில் உள்ளதை அறபு தெரியாதோரும் அறிந்து கொள்ள இலகுவாகும். அல்-குர்ஆன், நபிமொழி, சீறா ஆகியவற்றின் கருத்துக்களை உருது மற்றும் அதுவல்லாத ஏனைய மொழிகளுக்கு மொழிபெயர்ப்புச் செய்ததில் இந்திய உபகண்டத்திலுள்ள முஸ்லிம்களுக்கு பாரிய பங்குண்டு" (Yasin, 2021).

\section{அறபு மொழி இஸ்லாமிய இலக்கியங்களை மொழிபெயர்க்கையில் ஏற்பட்ட சிக்கல்கள்}

அறபு மொழி இலக்கியங்களை தமிழுக்கு மொழிபெயர்க்கும் போது பல்வேறு சிக்கல்களை மொழிபெயர்ப்பாளர் எதிகொள்ள வேண்டியுள்ளது. "அறபியிலிருந்து தமிழுக்கு மொழிபெயர்ப்புச் செய்யும் மொழிபெயர்ப்பாளர் அவை இரண்டும் இரு வேறுபட்ட மொழிக்குடும்பங்களைச் சேர்ந்திருப்பதால் சில பிரச்சினைகளை எதிர்கொள்கின்றார். அறபு மொழி செமித்திய மொழிக் குடும்பத்தைச் சேர்ந்தது. தமிழ் மொழி ஐரோப்பிய இந்திய (திராவிட) மொழிக் குடும்பத்தைச் சேர்ந்தது. எனவே, மொழிக்கட்டமைப்பு மற்றும் சேர்க்கை ரீதியாக ஒன்றுக்கொன்று முரண்பாடுகொண்டவை என்பதனால் அறபியிலிருந்து தமிழுக்கோ அல்லது தமிழிலிருந்து அறபுக்கோ மொழிபெயர்ப்புச் செய்வது இலகுவான காரியமல்ல. 
ஆகையால் மொழிபெயர்ப்பாளர், தனது மொழிபெயர்ப்புச் செயன்முறையின் போது பல்வேறுபட்ட பிரச்சினைகளை எதிர்கொள்கின்றார்" (Razick \& Fainas, 2015).

"ஒரு மக்கள் குழு அதன் சுற்றுப்புறங்களில் தன் பிழைப்பைத் தக்கவைத்துக் கொள்வதற்காக உருவாக்கிய வாழ்க்கை முறை, பழக்கவழக்கங்கள், நம்பிக்கைகள், பெறுமானங்கள், பழக்கவழக்கங்கள், அழகியல், சமூக நிறுவனங்கள் மற்றும் தகவல் தொடர்பு பாணிகளை உள்ளடக்கிய ஒரு வலைப்பின்னலே கலாசாரம் ஆகும். வேறு வார்த்தைகளில் கூறுவதானால், கலாசாரம் என்பது பல பரிமாண யதார்த்தத்தில் தங்கள் சொந்த அடையாளத்தை உருவாக்க வழிவகுக்கின்ற ஒரு தரிநபரை தனித்துவமாக்கும் பண்புகளை உள்ளடக்கியது" (Nagodawithana, 2020).

இதன்படி மொழிபெயர்ப்பின் போது ஒரு மொழிபெயர்ப்பாளர் மொழியை மட்டுமன்றி அது தாங்கி நிற்கும் கலாசாரத்தையும் சரர்த்தே மொழிபெயர்க்க வேண்டியுள்ளது. அறபு மொழி என்பது தொன்மையான வரலாற்றினைக் கொண்ட அறபு - இஸ்லாமியக் கலாசாரத்தினைக் கொண்டமைந்த மொழியாகும். அறபு மொழியையும் அதன் கலாசாரத்தையும் பிரிக்க முடியாத அளவு அவையிரண்டும் இணைந்துள்ளன. "அறபு இஸ்லாமிய கலாச்சாரத்தை உருவாக்குவதில் அறபு மொழி வலுவாக பங்களித்துள்ளது. உதாரணத்துக்கு, அறபு இஸ்லாமிய கலாச்சாரத்தை முன்னோக்கி வழி நடத்துவதில் அறபுமொழி முக்கிய பங்கு வகித்துள்ளது. அறபுமொழி கடந்த கால அம்சங்களை கவனத்தில் கொள்ளும் (Past-oriented) நோக்குடையது. அதனால்தான் அறபு இஸ்லாமிய கலாசாரம் கடந்த கால நோக்குடையதாக மாறியது. அறேபியர்கள் தமது தொர்பாடலுக்கு அறபு மொழிைப் பயன்படுத்துகின்றனர். இதனால், அறபு மொழி அவர்கள் மத்தியில் செல்வாக்குச் செலுத்துகின்றது. அத்துடன், முஸ்லிம்கள் அறபு மொழி வழியாக வெளிப்படுத்தப்பட்ட இஸ்லாத்தைப் பின்பற்றுவதன் காரணமாக அறபு மொழியால் கவரப்பட்டுள்ளனர்" (Ajami, 2016).

கலாசார ரீதியாக மொழி ஒன்று எதிர்கொள்ளும் பிரச்சினைகளில் மிக பிறமொழிச் சொற்களின் கலவையாகும். பிறமொழிச் சொற்கள் முழுவதுமாகவோ அல்லது பாதியளவிலோ நுழைந்து விடுகின்றன. எல்லா மொழிகளிலும் இந்நிலை காணப்படுகின்றது. மறைமலையடிகள் மொழியொன்றில் பிறமொழி கலப்பதற்குரிய காரணத்தை பின்வருமாறு விபரிக்கின்றார்; “ஓரு மொழியினைப் பேசும் மக்கள் தம் நாட்டையும் தம் இனத்தாரையும் விட்டு நீங்காமல் இருக்கும் வரையில், அவர் தாம் இருக்கும் நாட்டின் கண்ணே பிறமொழி பேசும் பிறநாட்டார் வந்து சேராதிருக்கும் வரையில், அவர் பேசும் மொழியில் அயல் மொழிச் சொற்கள் வந்து கலப்பதற்கு இடமேயில்லை. அங்ஙனமின்றி அவர் பல நாடுகளையும் அந்நாடுகளிலிலுள்ள பல திறப்பட்ட மக்களையும் போய்க் கண்டும், அவர் நாட்டுப் பண்டங்களை தாம் விலைகொண்டும், தம் நாட்டுப் பண்டங்களை அவர்க்கு (அவர்களுக்கு) விற்றும், அவர் தம் வழக்கவொழுக்கங்கள் சிலவற்றைத் தாங் கைப்பற்றியும், தமக்குரிய சிலவற்றை அவர் கைப்பற்றுமாறு தந்தும், ஒருவரது நாகரிகத்தை ஒருவர் பின்பற்றியும் ஒழுகும் உயர்ந்த அறிவும் உயர்ந்த நடையும் வாய்ந்தவர்களாயிருந்தால், அவர் பேசும் மொழியில் மற்ற மொழிச் சொற்கள் புகுந்து கலவாமல் இரா" (Maraimalaiyadihal, 2005).

தமிழை தாய்மொழியாகக் கொண்ட இஸ்லாமிய மக்கள் தமது அன்றாட வாழ்வியலில் அறபு மொழியைத் தவிர்க்க முடியாதுள்ளது. "முஸ்லிம்கள் தமிழ் மொழியை தாய் மொழியாகக் கொண்டவர்கள். ஆயினும் சமய ரீதியாக அனைத்து வணக்க வழிபாடுகளும் அறபு மொழியை அடிப்படையாகக் கொண்டவை. உதாரணமாக தினமும் ெய்யப்படும் ஐந்து நேரத் தொழுகையைக் குறிப்பிடலாம். அதில் ஓதப்படும் அனைத்து அம்சங்களும் அறபு மொழியில் அமைந்தவை. எனவே முஸ்லிம்கள் அறபு மொழியை விட்டு விலகிச் செல்ல முடியாதவாறு அதன் செல்வாக்கு காணப்படுகின்றது. இதனால் முஸ்லிம்கள் சிறுபராயத்திலிருந்தே அறபு மொழி கற்க ஆரம்பிப்பர். இது அவர்களது வாழ்வுடன் கலந்த ஒன்றாக உள்ளமை குறிப்பிடத்தக்கது" (Mohideen, 2018).

எனவே மொழிபெயர்ப்பாளர் இந்த மொழிக் காலாசரத்தை எதிர் கொள்ள வேண்டியுள்ளது. இலக்கு மொழிக்கு இத்தகைய சொற்களை மொழி பெயர்ப்பது பாரிய சிக்கலாகும். தமிழிலிலும் இதுவே நடைபெறுகின்றது. அறபியிலிருந்;து தமிழுக்கு மொழிபெயர்க்கும் போது சில அறபுச் சொற்களை அப்படியே எழுத வேண்டியுள்ளது. பெரும்பாலான முஸ்லிம்கள் தமது பேச்சுத் தமிழில் அச்சொற்களை 
அப்படியே பிரயோகிப்பர். சில ஆய்வாளர்களினால் அச்செற்கள் ‘அறபுத் தமிழ்' என்றும் அழைக்கப்படுகின்றன. உதாரணமாக, வுழு (தொழுகைக்காக செய்யப்படும் சுத்தம்), ஹஜ் (மக்கா சென்று நிறைவேற்றப்படும் கடமை), ஜகாத் (பொருளாதார ரீதியான கடமையாகச் செய்யப்படும் தர்மம்), ஜனாஸா (இறந்த உடல்), பஜ்ர் (அதிகாலை தொழுகை), சலாம் (முகமன் கூறுதல்) போன்றவற்றை உதாரணமாகக் கூறலாம். மொழிபெயர்ப்பாளர் தனது மொழிபெயர்ப்புச் செயன்முறையின் போது இச்சொற்களை அப்படியே பயன்படுத்துகின்றார்.

கலாசார ரீதியாக மொழிபெயர்ப்பு எதிர் கொள்ளும் மற்றொரு பிரச்சினை சொற்கள் கொண்டுள்ள கருத்து சார்ந்ததாகும். •வ்வொரு மொழிக்கும் ஒவ்வொரு தனித்துவமான கலாசாரம் உண்டு. மொழிபெயர்ப்பாளர் குறித்த மொழியொன்றின் வார்த்தையை பிற மொழிக்கு மொழிமெயர்ப்புச் செய்யும் போது வார்த்தைக் கலாசாரத்தை சிறப்பாக செய்ய முடியாது. மூல மொழிச் சொல்லின் எண்ணக் கருவையே இலக்கு மொழிக்கு அவரால் கொண்டு செல்ல முடியும். இந்த முரண்பாடான நிலைகள் சிறிய அளவிலிருந்து பெரிய அளவிற்கு இடம்பெறுகின்றன. மூல மொழியின் கலாசாரத்தைக் கவனத்தில் கொள்ளாமல் மொழிபெயர்க்கப்படுபவை வாசகனிடத்தில் மூல மொழியின் இயல்பு தொடர்பில் பிழையான விளக்கத்தை எற்படுத்தலாம். எனவே, மொழிபெயர்ப்பாளர் இ இரு மொழிகளினதும் கலாசாரத்தைப் புரிந்து கொள்ள வேண்டும். அப்போதுதான் உயிரோட்டமான மொழிபெயர்ப்பு உருவாகும். மூல மொழியின் ஒழுங்கமைப்பிற்கேற்ப மொழிபெயர்ப்பாளர் மொழிபெயர்த்தால் அ அது உயிரோட்டமிக்க மொழிபெயர்ப்பாக ஆகாது. பழமொழிகள், பொன்மொழிகள் இதற்குள் அடங்கும்" (Razick \& Fainas, 2015).

அறபு மொழியிலிருந்து தமிழுக்கு மொழிபெயர்ப்புச் செய்பவர் எதிர்கொள்ளும் மிக முக்கிய பிரச்சினைகளில் மற்றுமொன்று அறபியில் அமைந்துள்ள வணக்க வழிபாடுகள் சார்ந்த சொற்றொடர்கள் (மந்திரங்கள்) ஆகும். இஸ்லாத்தைப் பொருத்தவரை வணக்க வழிபாடுகளில் உச்சரிக்கப்படும் இத்தகைய சொற்றொடர்கள் மொழிபெயர்க்க முடியாதவை. ஆயினும் இவற்றின் கருத்துக்களை நேரடியாக மொழியெர்க்க முடியாத சிக்கல் மொழிபெயர்ப்பாளர்களுக்கு உண்டு. இதனால் தான் பெரும்பாலும் மொழிபெயர்ப்பின் போது அவை தமிழில் அறபு ஒலியன்களைக் கொண்டே எழுதப்படும். “இத்தகைய மொழிபெயர்ப்புக்கள் வாசகனிடம் எப்போதும் மர்மமாகவே இருக்கும். இவை மொழிபெயர்ப்பில் உள்ள தவறுகளாகும்" (Razick \& Fainas, 2015).

\section{முடிவுரை}

மொழிபெயர்ப்பு மனிதகுலம் கண்டுபிடித்த மிக முக்கியமான ஆக்கபூர்வமான பணிகளில் ஒன்றாகும். உலக வரலாற்றில் தோன்றிய பல கலாசாரப் பின்புலங்களை கொண்ட அனைத்து மக்களும் மொழிபெயர்ப்பில் ஈடுபட்டனர் தமது மொழியின் மற்றும் கலாசாரத்தின் தனித்துவத்தைப் பாதுகாத்த அதேவேளை அந்த கலாசாரத்தில் பிரதிபலன்களை ஏனைய சமூகங்கள் கண்டடையவும் வழிசெய்தன. ஒவ்வொரு கலாசாரத்தவர்களும் நமது கலாசாரத்தில் இல்லாத புதிய பல வி யங்களை ஏனைய மொழி கலாசார பின்புலத்திலிருந்து பெற்றுக்கொள்வதற்கு மொழிபெயர்ப்பு ஒரு கருவியாகக் கையாளப்பட்டது. இஸ்லாமிய வரலாற்றில் உமையாக்களின் ஆட்சி காலத்தில் கிரேக்க பிரதேசத்திலிருந்து அறிவுக் கருவூலங்கள் அறபு மொழிக்கு மொழிமாற்றம் செய்யப்பட்டன. நவீன ஐரோப்பா உருவாகவும், உலகின் விஞ்ஞான, கைத்தொழில், தொழில்நுட்ப புரட்சி ஏற்படவும் இதுவே காரணமாகிறது. இந்திய உபகண்டத்தில் வாழுகின்ற குறிப்பாக இந்தியா, இலங்கை போன்ற நாடுகளில் வாழும் தமிழ் பேசும் இஸ்லாமியர்கள் தமது சமயத்தின் பிரதான நூலாகிய அல்குர்ஆன் மற்றும் அல்ஹதீஸ் உள்ளிட்ட சமய நூல்களை அறபு மொழியிலிருந்து தமிழுக்கு மொழிபெயர்ப்பதில் ஆர்வம் காட்டினார். இவ்வாறு இருவேறுபட்ட மொழிக் குடும்பங்களைச் சேர்ந்த மொழிகளுக்கு இடையில் நடந்த இந்த மொழிபெயர்ப்பு செயல்முறையின் போது மொழிபெயர்ப்பானது பல்வேறு சவால்களை சந்திக்க நேர்ந்தது. குறிப்பாக பிறமொழிச் சொற்களின் கலப்பு, வட்டாரச் சொற்களின் செல்வாக்கு போன்ற மொழியியல்சார் பிரச்சினையும் மூல மொழியின் கலாசாரம் சார்ந்து நிற்கவேண்டுமென்ற நிலையும் குறிப்பிடத்தக்கவை. அதனை தீர்ப்பதற்கும் அந்த மொழிபெயர்ப்பாளர்கள் முயற்சி செய்தனர். இவ்வாய்வின் மூலம் 
மொழிபெயர்ப்பில் கலாசாரத்தை கட்டியெழுப்புவதன் அவசியம், கலாசாரங்களுக்கிடையிலான

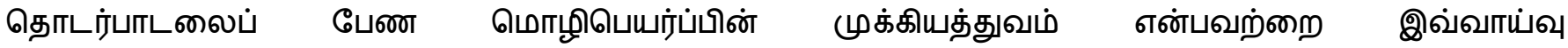
தெளிவுபடுத்தியுள்ளதுடன், தமிழுக்கு அறபியில் இருந்து மொழிபெயர்ப்பு செய்யும் பொழுது ஏற்படும் சிக்கல்களைக் கண்டறிந்து தீர்ப்பதற்கான வழிமுறைகளையும் விளக்கியுள்ளது.

\section{References}

Ajami, H., (2016) Arabic Language, Culture, and Communication, International Journal of Linguistics and Communication, 4 (1)120-123. https://doi.org/10.15640/ijlc.v4n1a12

Ashraff, AFM, (2010) Translation and its importance, Kalam Research Journal of Faculty of Arts \& Culture, Volume: IV, South Eastern University of Sri Lanka, Oluvil, Sri Lanka.

Mohamed Ashraff, AF., \& Ibrahim, T., (2013) Identify the Contribution and Necessity of Translation Practice for Construct the Social Harmony in Sri Lanka, Proceedings of the Third International Symposium, South Eastern University of Sri Lanka, Sri Lanka.

Chithambaram, C, (2019) Molipeyarppu ennum ariya kalai, Ulagattamil: An International Tamil Research Journal, 2(6) 1-6. https://www.researchgate.net/publication/339815697

Maraimalaiyadihal, (2005) Thamilil Pira Moli Kalappu, Paari Nilayam, India.

Mohideen, HLM. (2018) Influence of Arabic Language in Sri Lankan Tamil Short Stories - A special reference with Oddamawadi Arafath's Stories, Proceeding of $5^{\text {th }}$ International Symposium of FIA-2018, South Eastern University of Sri Lanka, Oluvil, Sri Lanka. http://ir.lib.seu.ac.lk/handle/123456789/3421

Nagodawithana, K.A., (2020) Culture in Translation: A Comprehensive Study, Journal of Social Sciences and Humanities Review, 5(4) 210-224.

Razick, AM. \& Fainas, ACF, (2015) The Problems Faced by Sri Lankan Translators From Arabic Language Into Tamil Language, Proceedings $5^{\text {th }}$ International Symposium,South Eastern University of Sri Lanka, Oluvil, Sri Lanks.

Vealyutham, S. S, (1985) Translation, International Institute of Tamil Studies, Chennai, India.

Yasin, MHBM., (2021), Dawabit Altarjamat Alsahihat Linusus Alsunat Walsiyrati, Maktabat Alnuwr Alkhayriati, http://www.noorsa.net/file.php?f=33681

Funding: No funding was received for conducting this study.

Conflict of Interest: The Author has no conflicts of interest to declare that they are relevant to the content of this article.

\section{About the License:}

\section{(c) (1)}

Attribution 4.0 International (CC BY 4.0)
(C) The Author 2022. The text of this article is open access and licensed under a Creative Commons Attribution 4.0 International License 